\title{
Probabilistic Tractography Using Q-Ball Modeling and Particle Filtering
}

\author{
Julien Pontabry and François Rousseau \\ LSIIT, UMR 7005 CNRS-Université de Strasbourg
}

\begin{abstract}
By assuming that orientation information of brain white matter fibers can be inferred from Diffusion Weighted Magnetic Resonance Imaging (DWMRI) measurements, tractography algorithms provide an estimation of the brain connectivity in-vivo. The two key ingredients of tractography are the diffusion model (tensor, high-order tensor, Q-ball, etc.) and the way to deal with uncertainty during the tracking process (deterministic vs probabilistic). In this paper, we investigate the use of an analytical Q-ball model for the diffusion data within a well-formalized particle filtering framework. The proposed method is validated and compared to other tracking algorithms on the MICCAI'09 contest Fiber Cup phantom and on in-vivo brain DWMRI data.
\end{abstract}

\section{Introduction}

In the last decade, Magnetic Resonance Imaging (MRI) has become a popular and powerful tool for medical imaging and brain understanding. In particular, Diffusion Weighted MRI (DWMRI) is a non-invasive imaging system, which gives information on water diffusion in human brain. These indirect observations of the white matter geometry in-vivo [3] allow nerve fiber reconstruction by using tractography algorithms. The two keypoints of a tractography algorithm are the diffusion model and the mathematical framework describing the tracking process.

Water diffusion is historically modeled by tensors [3], which has been quite successful in brain region with homogeneous intra-voxel fiber orientation. However, Berhens et al. have detected complex fiber architecture in approximately a third of voxels of the brain [4]. The standard tensor model does not handle such fiber bundle geometry well. A way to deal with this issue is to use High Angular Resolution Diffusion Imaging (HARDI) with appropriate diffusion models such as Q-ball or high-order tensors (HOT).

The second keypoint of tractography concerns the tracking process modeling. Tractography methods can be mainly categorized into two classes: deterministic and probabilistic. Deterministic solutions are subdivided into two groups: streamline and optimization based. In the first one, at each voxel, next direction(s) of fiber paths is(are) locally determined by mean direction(s) indicated by the diffusion model [142]. Such algorithms consist in a step-by-step construction of the solution. As only local mean directions are followed, there is a possible accumulation of errors during the tracking process. Optimization-based methods treat the tractography as an energy minimization problem [1618]. The purpose of these algorithms is to find the global solution, i.e. the solution that minimizes errors according to the diffusion model. 
Nevertheless, noise in MRI [11], partial volume effect [1] and possible ambiguity induced by the diffusion model can lead to uncertainty in the tracking process. Since this uncertainty may be represented in the form of probability density functions (PDF), probabilistic framework represents an attractive option for brain tractography [15]13:9.4]. A way to produce probabilistic maps of fibers consists in applying a streamline propagation process with directions randomly sampled from the PDF. The fraction of these sampled paths passing through each voxel provides a stochastic measure of the strength of connectivity between that voxel and the seed points. In such framework, a keypoint is the effectiveness of the sampling stage [15|20]. In particular, as fiber paths can be modeled as Markov chains, sequential Monte Carlo and Markov Chain (MCMC) methods can be efficiently used for the sampling stage [20].

Extending previous work of Zhang et al. [20], we investigate the use of Q-ball based modeling within a particle filtering framework. The reliability of Q-Ball models allows us to extract more information from data, such as the orientation distribution function (ODF) which provides a precise idea of the underlying fiber architecture at every voxel. Then, a non-linear state-model is used for the tracking modeling and the probabilistic maps of fibers are estimated using a particle filtering algorithm.

\section{Proposed Method}

\subsection{Diffusion Model}

Let consider an image domain $\Omega \subset \mathbb{R}^{3}$ and diffusion weighted measurement in $N$ directions. The diffusion signal is decomposed into spherical harmonics by linear regression [6]. Then, applying Funk-Radon transform to the signal decomposition, the diffusion ODF can be analytically computed at point $x \in \Omega$ and in direction $u=(\theta, \phi)[6]$. In order to get a sharper ODF, the fiber orientation distribution function (fODF) $\psi_{x}(\theta, \phi)$ is computed from diffusion ODF by a spherical deconvolution [7]. Let the estimated diffusion signal $S_{x}$ and the fODF $\psi_{x}$ be the diffusion observations at $x \in \Omega$.

\subsection{Fiber Tracking Model}

In space state model, a fiber path $v_{0: k}$ can be modeled as a sequence of vectors in a volume $\Omega$ and can be built iteratively. Fiber paths are assumed to have Markovian nature. From a given starting point $x_{0} \in \Omega$, points of path $v_{0: k}$ are defined as $x_{k+1}=x_{k}+\lambda v_{k}$, where $\lambda \in \mathbb{R}$ is the step size which is assumed to be constant and $v_{k}$ is a unit vector.

The principle of particle filtering is to sample sequentially a set of $M$ paths from the starting point $x_{0} \in \Omega$, given observations $z_{k}=\left\{S_{x_{k}}, \psi_{x_{k}}\right\}$. This means that $M$ particles are placed at point $x_{0}$ at step $k=0$ and are propagated as time progresses. Given the set of particles $\left\{v_{0: k}^{(m)}, w_{k}^{(m)}\right\}_{m=1}^{M}$ at step $k$, the propagation to the next step $k+1$ is performed following three stages: prediction, weighting and selection.

In prediction stage, since the posterior $p\left(v_{0: k} \mid z_{1: k}\right)$ cannot be evaluated, the importance density $\pi\left(v_{0: k} \mid z_{1: k}\right)$ (which is an approximation of the posterior density) is be used to simulate each of paths' vectors. At step $k$ and considering Markovian nature of fiber paths, the vector $v_{k}$ of a path $v_{0: k-1}$ is sampled according to the recursive form of importance density [8]: $\pi\left(v_{k} \mid v_{0: k-1}, z_{1: k}\right)$. 
After prediction stage, a weighting stage giving a measurement of reliability of the approximation of the posterior density is performed. A particle's weight is written recursively as [8]

$$
\tilde{w}_{k}^{(m)} \propto \tilde{w}_{k-1}^{(m)} \frac{p\left(z_{k} \mid v_{k}^{(m)}\right) p\left(v_{k}^{(m)} \mid v_{k-1}^{(m)}\right)}{\pi\left(v_{k}^{(m)} \mid v_{0: k-1}^{(m)}, z_{1: k}\right)},
$$

where $\tilde{w}_{t}^{(m)}$ are normalized weights, $k$ is the current state, $p\left(z_{k} \mid v_{k}^{(m)}\right)$ and $p\left(v_{k}^{(m)} \mid v_{k-1}^{(m)}\right)$ are respectively likelihood and prior densities.

Choosing recursive importance distribution leads to an increasing variance of the weights as the time progresses [12]. The purpose of the final stage selection is to avoid this degeneracy. We first measure degeneracy of the cloud of paths using the effective sample size (ESS): $N_{\text {ESS }}=1 / \sum_{m=1}^{M} \tilde{w}_{(m)}^{2}$. When $N_{\text {ESS }}$ decreases below a fixed threshold $\varepsilon_{\mathrm{ESS}}$, a resampling procedure is used in order to eliminate particles with low weight [8].

\subsection{Densities}

The Prior Distribution. As in [20], the von Mises-Fisher (vMF) distribution has been selected as prior because it is a parametric distribution for directional data. This distribution is parametrized by $\mu$ and $\kappa$, respectively called mean direction and concentration. The greater the value of $\kappa$, the stronger is the concentration of the distribution around mean direction $\mu$. Considering the tractography problem and the tracking model, the value of concentration parameter $\kappa$ is a smoothness constraint of fiber path.

The Importance Density. The iterative solution estimation relies on the approximation of the posterior, i.e. on the importance density. At each step, knowing a path's vector sequence and its observation information, next direction added to path is sampled according to the importance density. The optimal importance density is $p\left(v_{k} \mid v_{k-1}, z_{1: k}\right)$, because conditionally upon $v_{k}$ and $z_{1: k}$, the variance of the importance weights $w_{k}$ is then minimal [8]. Since it is difficult to sample from $p\left(v_{k} \mid v_{k-1}, z_{1: k}\right)$, a usual choice is to use for importance the same distribution as prior. Nevertheless, since no observation information is used, generated particles using the prior are often outliers of the true posterior distribution. As importance function, we choose a local linearization using observations. The ODF functions model water diffusion in the brain and give an estimation of the underlying fiber architecture. Each ODF's maxima should indicate fibers' directions. Let $\Lambda_{k}$ be a set of directions where $\psi_{x_{k}}$ is locally maximum. Then, importance density is defined in dimension 3 as vMF mixture:

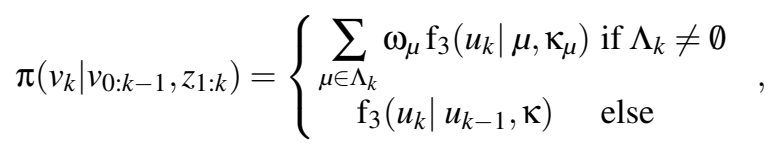

where $f_{3}$ is the vMF on the 2 -sphere in $\mathbb{R}^{3}, u_{k}$ is the unit vector in spherical coordinates corresponding to the unit vector $v_{k}$ in Cartesian coordinates, $\kappa_{\mu}$ is the concentration depending on observations, $\kappa$ is the concentration parameter and $\omega_{\mu}$ are mixture proportions such that $0 \leq \omega_{\mu} \leq 1$ and $\sum_{\mu \in \Lambda_{k}} \omega_{\mu}=1$. Each $\omega_{\mu}$ is proportional to the ODF value in direction $\mu$. 
In [20], the concentration parameter of importance is computed as an exponential function of fractional anisotropy (FA). For ODF functions, FA in one direction can be measured as the mean curvature of the function in that direction [5]. Thus, the concentration parameter $\kappa_{\mu}$ is proportional to ODF curvature in direction $\mu: \kappa_{\mu} \propto H(\mu)$ where $H(\mu)$ is the mean curvature of $\psi_{x_{k}}$ in direction $\mu$. The use of a vMF mixture allows us an efficient sampling procedure of the importance distribution [19].

This definition of importance distribution approximate likelihood distribution [20]. The paths cloud is then weighted by importance weights proportionally to prior distribution. This means that each particle is projected backward in order to know its probability to have a predecessor among paths cloud at previous step.

The Likelihood. Partially due to noise, there is uncertainty in MRI diffusion information. The observation density is supposed to be a measure of this uncertainty. In term of probabilities, likelihood defines a measure of how observations match the current model. Therefore, we model likelihood density as a distance error between measured observations and observations matching perfectly the current state model. At step $k$, $u_{k}$ is the direction sampled according to the importance and $\mu$ is assumed to be the mean vector of the sampled vMF distribution (in order to get a direction $u_{k}$ ). According to section 2.1, the diffusion signal at position $x_{k}$ is described by $S_{x_{k}}$. Let $\bar{S}_{x_{k}}$ be the diffusion signal at position $x_{k}$ if the sampled direction $u_{k}$ was exactly the mean direction $\mu$, i.e. $\mu=u_{k}$. Then, $\bar{S}_{x_{k}}$ is determined from $S_{x_{k}}$ by a rotation of angle $\widehat{\mu u_{k}}$ in three dimensional space: $\bar{S}_{x_{k}}=\operatorname{rot}\left(\widehat{\mu u_{k}}, S_{x_{k}}\right)$, where $\widehat{\mu u}_{k}$ denotes the angle between $\mu$ and $u_{k}$.

Similary to [20], let consider $\bar{S}_{x_{k}}$ as the diffusion signal and the MRI intensities $\mathcal{U}_{x_{k}}$, a noisy measure of it, i.e. $\mathcal{U}_{x_{k}}=\bar{S}_{x_{k}}+\varepsilon$. Noise in MRI images can be described closely approximated for moderate-large SNR by a normal distribution [11] with a zero-mean and standard deviation of noise in MRI diffusion weighted image, i.e. $\varepsilon=$ $\mathcal{U}_{x_{k}}-\bar{S}_{x_{k}} \sim \mathcal{N}\left(0, \Sigma^{2}\right)$. The standard deviation of diffusion MRI image in each gradient directions $\sigma_{i}$ is estimated by least square estimation and pseudo-residuals [10]. Thus, likelihood is written as a multiplication of error distribution in each gradient direction $g_{i}$ :

$$
p\left(z_{k} \mid v_{k}\right)=\prod_{i=1}^{N} \mathcal{N}\left(\mathcal{U}_{x_{k}}\left(g_{i}\right)-\bar{S}_{x_{k}}\left(g_{i}\right) \mid 0, \sigma_{i}^{2}\right) .
$$

\section{Results}

The tractography method has been evaluated on the MICCAI'09 Fiber Cup phantom and on in-vivo brain DWMRI data. Since the sampled particles evolve in a continuous domain and data is acquired in a discrete manner, a third-order B-Spline interpolation is applied to dataset when required. Each tractography is performed by sampling 1000 particles. The result of the algorithm is an estimation of the posterior density which corresponds to a connectivity map. Fiber bundles can be obtained by estimating the maximum a posteriori (MAP) of the posterior density. 


\subsection{Fiber Cup}

The Fiber Cup is a tractography contest proposed at the MICCAI conference help in London in 20091. By containing several crossing, kissing, splitting and bending fiber configurations, this phantom is a very convenient way to compare the proposed method with other existing approaches. The Fiber Cup phantom size is $64 \times 64 \times 3$ voxels with a resolution of $3 \times 3 \times 3 \mathrm{~mm}$. It contains 2 repetitions of 65 gradient directions each, including 1 baseline direction for each repetition. The b-value is 1500 for each direction. The seeds' locations of the contest are exposed in Fig. 1.

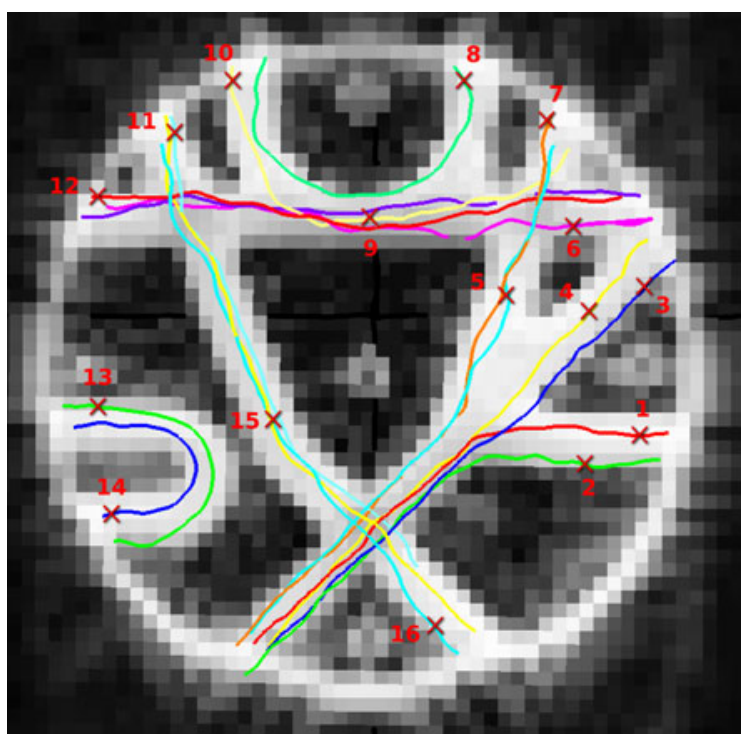

Fig. 1. Fibers estimations of our algorithm on the Fiber Cup phantom. Seeds are marked with red cross.
Table 1. Quantitative results of the proposed algorithm on the Fiber Cup phantom. When the proposed algorithm reaches the first three places of the contest, the corresponding results are marked in bold.

\begin{tabular}{c||c|c|c}
\hline & L2 & tan & curv \\
\hline \hline F1 & $\mathbf{1 . 6 8 9 5 2}$ & 14.8774 & 0.155911 \\
\hline F2 & $\mathbf{2 . 9 3 3 8 5}$ & 25.9358 & 2.81907 \\
\hline F3 & $\mathbf{1 . 6 4 9 5 1}$ & 13.8554 & 0.165466 \\
\hline F4 & $\mathbf{2 . 1 2 3 8 5}$ & 16.8663 & 0.157994 \\
\hline F5 & $\mathbf{2 . 7 2 5 9 8}$ & $\mathbf{1 6 . 3 2 5 9}$ & 0.160928 \\
\hline F6 & $\mathbf{3 . 5 1 5 3 7}$ & 23.713 & 1.02897 \\
\hline F7 & $\mathbf{3 . 5 8 3 1 3}$ & $\mathbf{2 1 . 2 4 5}$ & 2.54886 \\
\hline F8 & $\mathbf{2 . 3 4 6 2 4}$ & 21.7216 & 3.82264 \\
\hline F9 & $\mathbf{3 . 0 3 3 5 8}$ & 17.6071 & 0.150982 \\
\hline F10 & 8.96364 & 31.2289 & 0.147886 \\
\hline F11 & 3.53476 & 17.6234 & 0.136861 \\
\hline F12 & $\mathbf{3 . 8 7 7 7 3}$ & $\mathbf{2 0 . 7 6 7 2}$ & 0.183536 \\
\hline F13 & $\mathbf{2 . 6 0 1 6 8}$ & 16.2984 & 0.17025 \\
\hline F14 & $\mathbf{2 . 4 7 0 2 6}$ & $\mathbf{1 6 . 2 0 7 9}$ & 0.133395 \\
\hline F15 & $\mathbf{2 . 2 4 9 2 5}$ & 18.6442 & 0.157172 \\
\hline F16 & $\mathbf{4 . 2 6 7 3 6}$ & 17.4609 & 0.136369 \\
\hline & \multicolumn{3}{|c}{}
\end{tabular}

The quantitative results of our algorithm are summarized in the Tab. 1 and estimated fibers from the particles cloud are shown in Fig 1 . The algorithm gets 35 points according to the Fiber Cup contest and would be at the $3^{\text {rd }}$ position in the ranking. Considering only the spatial metric, our algorithm places itself to the first position with 31 points, before [17] which gets 22 points. As shown in Table 1] although the method has pleasing results with the spatial metric (L2 norm), it suffers from a lack of precision in both tangent and curvature metric. This might be due to the quality of the estimation of the MAP of the posterior density and not to the particle filtering algorithm itself. Nevertheless, satisfactory results are generated in a short computation time. For instance, the

\footnotetext{
${ }^{1}$ The website http://www.Inao.fr/spip.php?rubrique79 provides details and results about this competition. The phantom used to compare tractographies and the comparison program are also available on this website.
} 


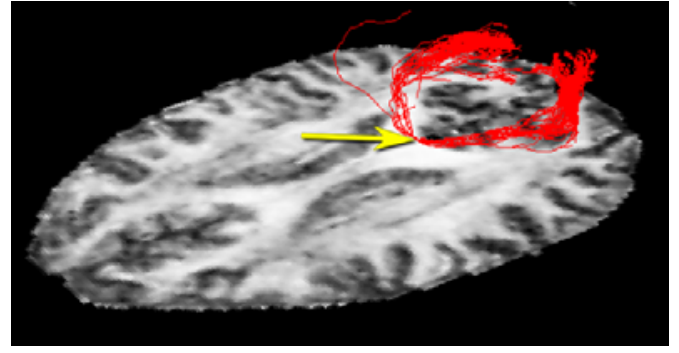

(a) Particles cloud

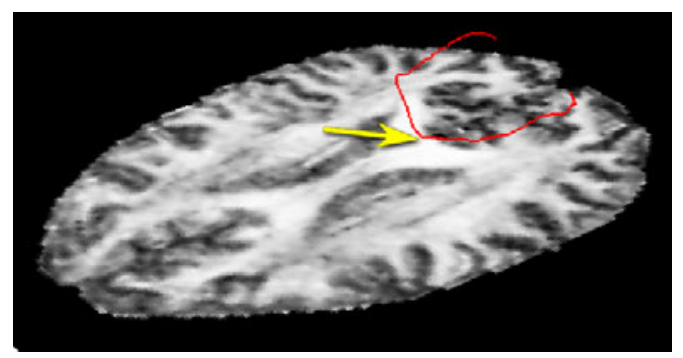

(b) MAP estimate of the posterior density

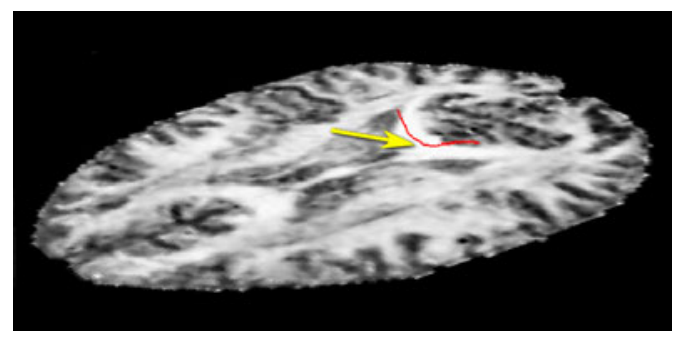

(c) fODF-STR result [7]

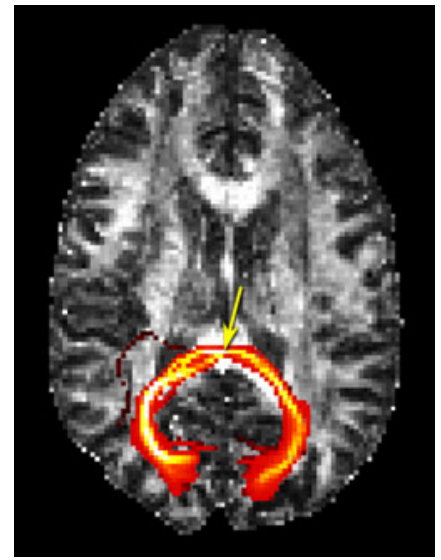

(d) Connectivity map

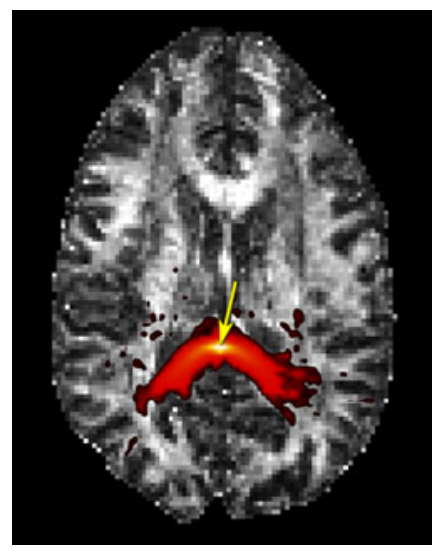

(e) fODF-PROBA result [7]

Fig. 2. Results of tractographies of adult human brain starting from a seed point in the corpus callosum indicated by a yellow arrow. Each result is displayed on a FA image slice. Tractographies were performed using fODF-STR (Fig. 2(c)], fODF-PROBA (Fig. 2(e)] and the proposed algorithm (Fig.2(a) 2(b) and 2(d).

winner of this contest [17] uses a global algorithm which performed the tractography in one day of computation time whereas ours implementation did run in 8 minutes on a computer with 8 processor Intel Xeon $2.4 \mathrm{GHz}$ (including the preprocessing, i.e. the model estimation, the noise estimation, the maxima extraction, etc.).

\subsection{In-vivo Data}

In-vivo brain dataset comes from the community MIDAS / National Alliance for Medical Image Community (NAMIC) 2 and was acquired from a healthy adult volunteer

\footnotetext{
${ }^{2}$ http://insight-journal.org/midas/collection/view/190
} 
using a 3 Tesla GE system. It contains a $144 \times 144 \times 85$ volume image with $1.7 \times$ $1.7 \times 1.7 \mathrm{~mm}$ voxel resolution. The diffusion signal was measured in 51 directions with $b=900 \mathrm{~s} . \mathrm{mm}^{-2}$ and there are 8 baseline images $\left(b=0 \mathrm{~s} . \mathrm{mm}^{-2}\right)$. During the model estimation, the 8 baseline images have been used by averaging them. The model order for the spherical harmonics is $l=4$. The proposed algorithm was applied to this volume image with a step length of $0.85 \mathrm{~mm}$, a resampling threshold $\varepsilon_{\mathrm{ESS}}=60 \%$ and 1000 particles. The tractography has been performed on a brain mask defined by voxels with a fractional anisotropy (FA) value greater than 0.2 . In order to show the contribution of the particle filtering framework into the tractography results, the proposed algorithm is compared with two other tractography frameworks (using the same diffusion model): fODF streamline (fODF-STR) and fODF "probabilistic" (or random walk, named later fODF-PROBA) [7].

Figure 2 shows the results of a tractography starting from a seed point in the corpus callosum. An axial slice of FA map is shown as a reference background image. The particle cloud is shown in Fig. 2(a) From this cloud, both fiber path and posterior density map (projected on the axial slice) have been generated (respectively Fig. 2(b) and 2(d)]. Using the same experimental parameters, our implementations of fODF based algorithms, were applied to the dataset (results are displayed respectively in Fig. 2(c) and 2(e). The propagation of fODF-STR is early stopped because it reaches the boundary of the brain mask, resulting in a partial tractography (Fig. 2(c)]. The fODF-PROBA algorithm leads to a very smooth posterior density approximation (Fig. 2(e) due to the diffusion-based random walk strategy used in this algorithm.

\section{Discussion}

In this article, a probabilistic tractography method has been presented based on the well-formalized particle filtering framework and using an analytical Q-ball model for diffusion data. The output of the algorithm is an estimate of the posterior density of the white matter fibers. The keypoints of the method are the three densities: prior, likelihood, importance. First, the prior density constrains the solution. Then, the likelihood density ensures the reasonableness of the estimation with a noise model of DWMRI data. Finally, fast and efficient sampling is realized by a vMF mixture.

As shown by the experiments performed on the Fiber Cup phantom, the use of a Qball data modeling within the particle filtering framework leads to accurate estimations of complex fiber configurations. Experiments on in-vivo data have shown the contribution of the particle filtering framework compared to streamline approach or random walk like techniques. Further work could study the whole diffusion information available, without any diffusion model constraint. Indeed, techniques for estimating models induce approximations that can exaggerate uncertainty in the data.

Acknowledgment. The research leading to these results has received funding from the European Research Council under the European Community's Seventh Framework Programme (FP7/2007-2013 Grant Agreement no. 207667). 


\section{References}

1. Alexander, A.L., Hasan, K.M., Lazar, M., Tsuruda, J.S., Parker, D.L.: Analysis of partial volume effects in diffusion-tensor MRI. Magnetic Resonance in Medicine 45(5), 770-780 (2001)

2. Basser, P.J., Pajevic, S., Pierpaoli, C., Duda, J., Aldroubi, A.: In-vivo fiber tractography using DT-MRI data. Magnetic Resonance in Medicine 44(4), 625-632 (2000)

3. Basser, P., Mattiello, J., LeBihan, D.: MR diffusion tensor spectroscopy and imaging. Biophysical Journal 66(1), 259-267 (1994)

4. Behrens, T., Berg, H.J., Jbabdi, S., Rushworth, M., Woolrich, M.: Probabilistic diffusion tractography with multiple fibre orientations: What can we gain? NeuroImage 34(1), 144 155 (2007)

5. Bloy, L., Verma, R.: On computing the underlying fiber directions from the diffusion orientation distribution function. In: Metaxas, D., Axel, L., Fichtinger, G., Székely, G. (eds.) MICCAI 2008, Part I. LNCS, vol. 5241, pp. 1-8. Springer, Heidelberg (2008)

6. Descoteaux, M., Angelino, E., Fitzgibbons, S., Deriche, R.: Regularized, fast, and robust analytical q-ball imaging. Magnetic Resonance in Medicine 58(3), 497510 (2007)

7. Descoteaux, M., Deriche, R., Knosche, T., Anwander, A.: Deterministic and probabilistic tractography based on complex fibre orientation distributions. IEEE Transactions on Medical Imaging 28(2), 269-286 (2009)

8. Doucet, A., Godsill, S., Andrieu, C.: On sequential monte carlo sampling methods for bayesian filtering. Statistics and Computing 10(3), 197-208 (2000)

9. Friman, O., Farneback, G., Westin, C.: A bayesian approach for stochastic white matter tractography. IEEE Transactions on Medical Imaging 25(8), 965-978 (2006)

10. Gasser, T., Sroka, L., Jennen-Steinmetz, C.: Residual variance and residual pattern in nonlinear regression. Biometrika 73(3), 625-633 (1986)

11. Gudbjartsson, H., Patz, S.: The rician distribution of noisy mri data. Magnetic Resonance in Medicine 34(6), 910-914 (1995)

12. Kong, A., Liu, J.S., Wong, W.H.: Sequential imputations and bayesian missing data problems. Journal of the American Statistical Association 89(425)

13. Lazar, M., Alexander, A.L.: Bootstrap white matter tractography (BOOT-TRAC). NeuroImage 24(2), 524-532 (2005)

14. Mori, S., Crain, B.J., Chacko, V.P., Zijl, P.C.M.V.: Three-dimensional tracking of axonal projections in the brain by magnetic resonance imaging. Annals of Neurology 45(2), 265269 (1999)

15. Parker, G., Alexander, D.: Probabilistic monte carlo based mapping of cerebral connections utilising whole-brain crossing fibre information. In: Information Processing in Medical Imaging, p. 684695 (2003)

16. Parker, G., Wheeler-Kingshott, C., Barker, G.: Estimating distributed anatomical connectivity using fast marching methods and diffusion tensor imaging. IEEE Transactions on Medical Imaging 21(5), 505-512 (2002)

17. Reisert, M., Mader, I., Anastasopoulos, C., Weigel, M., Schnell, S., Kiselev, V.: Global fiber reconstruction becomes practical. NeuroImage 54(2), 955-962 (2011)

18. Staempfli, P., Jaermann, T., Crelier, G., Kollias, S., Valavanis, A., Boesiger, P.: Resolving fiber crossing using advanced fast marching tractography based on diffusion tensor imaging. NeuroImage 30(1), 110-120 (2006)

19. Ulrich, G.: Computer generation of distributions on the m-Sphere. Journal of the Royal Statistical Society. Series C (Applied Statistics) 33(2), 158-163 (1984)

20. Zhang, F., Hancock, E.R., Goodlett, C., Gerig, G.: Probabilistic white matter fiber tracking using particle filtering and von Mises-Fisher sampling. Medical Image Analysis 13(1), 5-18 (2009) 